\title{
Orthogonal cartograms with few corners per face *
}

\author{
Therese Biedl $^{\dagger} \quad$ Lesvia Elena Ruiz Velázquez ${ }^{1}$ \\ Technical Report CS-2010-21
}

\begin{abstract}
We give an algorithm to create orthogonal drawings of 3-connected 3-regular planar graphs such that each interior face of the graph is drawn with a prescribed area. This algorithm produces a drawing with at most 12 corners per face and 4 bends per edge, which improves the previous known result of 34 corners per face.
\end{abstract}

${ }^{*}$ Research supported by NSERC.

${ }^{\dagger}$ Cheriton School of Computer Science, University of Waterloo, Waterloo, ON N2L 3G1, Canada, \{biedl, leruizve\}@uwaterloo.ca. 


\section{Introduction}

A planar graph is a graph that can be drawn without crossing. Fáry, Stein and Wagner [10, 23, 27] proved independently that every planar graph has a drawing such that all edges are drawn as straight-line segments.

Sometimes additional constraints are imposed on the drawings. The most famous one is to have integer coordinates while keeping the area small; it was shown in 1990 that this is always possible in $O\left(n^{2}\right)$ area [7,22]. Another restriction might be to ask whether all edge lengths are integral; this exists if the graph is 3-regular [11], but is open in general.

We consider drawings with prescribed face areas. Ringel [20] showed that not all planar graphs have straight-line drawings with prescribed face areas. Thomassen [24] showed that they do exist for planar graphs with maximum degree 3. We study here orthogonal drawings.

Graph drawings with prescribed face areas are motivated by cartograms, which are distortions of maps such that faces (i.e., countries in a map) should be proportional to some property of the country, such as population. Note that maps, with a few exceptions, have maximum degree 3 (i.e., no four countries meet in a point.) We use the term orthogonal cartogram for an orthogonal drawing with prescribed areas for interior faces.

Known results Raisz introduced rectangular cartograms [19], which are orthogonal cartograms where every face (including the outer-face) is a rectangle. Kant and He [14] and Ungar [25] characterized (independently) exactly which planar graphs have a rectangular drawing, but not all these graphs have a rectangular drawing that respects given face areas. Eppstein et al. [9] gave an algorithm to test whether a graph has a rectangular cartogram for all possible area assignments, but their algorithm is not polynomial.

So to create a cartogram of an arbitrary graph, one must either allow error on the area, or on the adjacencies, or allow more complicated shapes. In 2004, van Kreveld and Speckmann gave an algorithm to draw rectangular cartograms, where there can be a small error both on the adjacencies and on the areas [26]. In 2005, de Berg, Mumford, and Speckmann proved that any planar graph with maximum degree 3 and 3-connected dual can be drawn orthogonally with prescribed face areas and at most 60 corners per face [3]. In their journal version they reduced this to 40 corners [5], and Kawaguchi and Nagamochi reduced it further to 34 corners per face [15]. In a different work, Rahman, Miura and Nishizeki, gave an algorithm to draw a special class of graphs called good slicing graphs with at most 8 corners per face [18]. See also $[4,6,12,16]$ for other works on rectangular and orthogonal cartograms.

Our results In this paper, we give a new algorithm to create orthogonal cartograms of 3-connected 3 -regular planar graphs. The resulting drawings have at most 12 corners per face. The outer-face is a rectangle. Our work hence improves the work by de Berg et al. and Kawaguchi and Nagamochi $[5,15]$. Contrasting our work to the one by Rahman et al. [18], they use fewer corners, but our result is significantly less restrictive on the graph class. Our drawings can be found in $O(n \log n)$ time.

Our approach is substantially different from the one in [3]. They obtained drawings by modifying the graph until it has a rectangular cartogram, and then "fixing up" adjacencies by adding thin connectors where needed. Our approach works throughout with the original graph and produces a drawing directly. We find this a more natural approach, and it also eases bounding the number of bends. Our algorithm is based on Kant's canonical ordering [13], a useful tool for many graph drawing algorithms. However, it does not use the ordering directly, but uses it to split the graph 
into two smaller graphs (or one graph and a face); this decomposition to our knowledge was not known before and may be of independent interest in the graph drawing community.

Due to space limits we omit some details of correctness; a full exposition can be found in [21].

\section{Background and Preliminaries}

Let $G=(V, E)$ be a graph with $n=n(G)=|V|$ vertices and $m=m(G)=|E|$ edges. Throughout this paper, we will assume that $G$ is simple (has no loops or multiple edges) and planar, i.e., can be drawn without crossing. Such a drawing determines faces, i.e., the maximal connected pieces of the plane. The unbounded piece in such a drawing is called the outer-face; we assume that the outer-face has been fixed.

An orthogonal drawing of a planar graph is a drawing without crossing where vertices are represented by points and edges are represented by sequences of contiguous horizontal or vertical line segments. In what follows, we will identify the graph-theoretic entity (vertex, edge) with the geometric entity (point, sequence of segments) that represents it. A place where an edge switches direction is called a bend.

For orthogonal drawings, it is important to keep the number of bends small, so that the drawings can be understood easily. We will bound here both the number of bends per edge, and the number of bends that are on a face. For cartogram purposes, it is also important to keep the shape of the face as simple as possible. Hence we will also bound the number of corners in a face, which is the number of angles other than $180^{\circ}$. Every bend is a corner, but corners may also occur at vertices.

Let $A$ be a function that assigns non-negative integers to interior faces of $G$. We say that a planar drawing of $G$ respects the given face areas if every interior face $f$ of $G$ is drawn with area $A(f)$. We use $A(G)$ to denote the sum of $A(f)$ over all interior faces $f$ of a graph $G$.

A graph is 3-connected if it is necessary to remove at least 3 vertices to make it disconnected. A planar 3-connected graph is known to have a canonical ordering [13]. This is a partition $V=$ $V_{1} \cup \ldots \cup V_{K}$ such that $V_{1}$ contains the endpoints $v_{1}, v_{2}$ of an edge on the outer-face, as well as exactly all vertices on the interior face adjacent to $\left(v_{1}, v_{2}\right)$. For $1<k<K$, set $V_{k}$ can be (a) a singleton $\{z\}$ that has at least two neighbours in $V_{1} \cup \ldots \cup V_{k-1}$ and at least one neighbour in $V_{k+1} \cup \ldots \cup V_{K}$, or (b) a chain, i.e., path $\left\{z_{1}, \ldots, z_{r}\right\}$ of $r \geq 2$ vertices such that $z_{1}$ and $z_{r}$ have exactly one neighbour in $V_{1} \cup \ldots \cup V_{k-1}$, no other vertex of the chain has a neighbour in $V_{1} \cup \ldots \cup V_{k-1}$, and all vertices of the chain have a neighbour in $V_{k+1} \cup \ldots \cup V_{K}$. Finally, $V_{K}$ is a singleton $\left\{v_{n}\right\}$ that belongs to the outer-face and is adjacent to $v_{1}$. See Figure 1 for an example, and many graph drawing papers and books (e.g. [13, 2, 1, 17, 8]) for more details on the canonical ordering and its applications.

We assume that the input graph is undirected, but during the algorithm we impose directions onto some of the edges, resulting in a partially oriented graph. In such a partially ordered graph, the in-degree (out-degree) of a vertex is the number of its incoming (outgoing) edges.

\section{Dart-shaped graphs}

Assume that $G=(V, E)$ is a 3-connected 3-regular planar graph with a canonical ordering $V=$ $V_{1} \cup \ldots \cup V_{K}$. This naturally implies a partial edge orientation of $G$ which we call a canonical orientation: direct the edges from a vertex in $V_{i}$ to a vertex in $V_{j}$ if $i<j$. Edges between vertices 
in the same set remain undirected. This partial orientation is acyclic, i.e., it contains no directed cycle.

In a partially oriented graph we use the term vertical path for a simple path where all edges are directed from one end to the other. In a canonical orientation a vertical path connects vertices in $V_{i_{1}}, V_{i_{2}}, \ldots, V_{i_{k}}$ with $i_{1}<i_{2}<\cdots<i_{k}$; hence the name. A horizontal path is a simple path where all edges are undirected. In a canonical orientation, all vertices in a horizontal path belong to the same set $V_{i}$; hence the name.

Definition 1 Let $G=(V, E)$ be a plane graph with an acyclic partial edge orientation. $G$ is called dart-shaped with respect to the orientation if:

D1. The outer-face consists of a horizontal path $P_{b}$ (connecting a vertex $c_{l}$ to a vertex $c_{r}$ ), and two vertical paths, $P_{l}$ (from $c_{l}$ to a vertex $c_{t}$ ), and $P_{r}$ (from $c_{r}$ to $c_{t}$ ). These paths are interior vertex-disjoint. The vertices $c_{l}, c_{r}, c_{t}$ will be called left, right and top corner-vertex, and the paths $P_{b}, P_{l}, P_{r}$ will be called bottom, left and right path of $G$.

D2. Walking around any face, we encounter an undirected edge, a non-empty vertical path in the same direction as the walk, a horizontal path (possibly empty), and a non-empty vertical path in the opposite direction to the walk.

D3. $\operatorname{deg}\left(c_{l}\right)=\operatorname{deg}\left(c_{r}\right)=\operatorname{deg}\left(c_{t}\right)=2$. All other vertices have degree at most 3 .

D4. Every vertex $\neq c_{t}$ has exactly one outgoing edge.

See also Figure 1b. From now on, whenever we speak of a dart-shaped graph $G$, we will use $c_{l}, c_{r}, c_{t}, P_{b}, P_{l}, P_{r}$ for its corner-vertices and paths without specifically recalling that notation. Notice that D3. and D4. imply that no vertex on $P_{b}$ has an incoming edge.

Using the properties of a canonical ordering, it is easy to see the following lemma.

Lemma 3.1 Let $G$ be a planar 3-connected 3-regular graph. Let $V_{1} \cup \ldots \cup V_{K}$ be a canonical ordering of $G$, with $V_{1}=\left\{v_{1}, \cdots, v_{j}\right\}$ (in order around the face) and $V_{K}=v_{n}$. Then $G^{\prime}=G-v_{1}$, with the partial orientation induced by the canonical orientation of $G$, is dart-shaped and its corner-vertices are $c_{l}=v_{j}, c_{r}=v_{2}$ and $c_{t}=v_{n}$.

\subsection{Decomposing a dart-shaped graph}

We now show how to split a dart-shaped graph into smaller dart-shaped graphs, which will allow us later to build a recursive algorithm to create orthogonal cartograms. The partial edge orientation remains the same throughout all splits, and hence will not always be mentioned.

Theorem 1 Let $G$ be a dart-shaped graph. If $G$ has more than one interior face, then:

(a) $G$ can be decomposed into two dart-shaped graphs by splitting along a vertical path from the interior of $P_{b}$ to the interior of $P_{l}$ or $P_{r}$ (see Figure 2a), or

(b) $G$ can be decomposed into a dart-shaped graph and a face containing $c_{l}$ and $c_{r}$ by splitting along a horizontal path from the interior of $P_{l}$ to the interior of $P_{r}$ (see Figure 2b). 


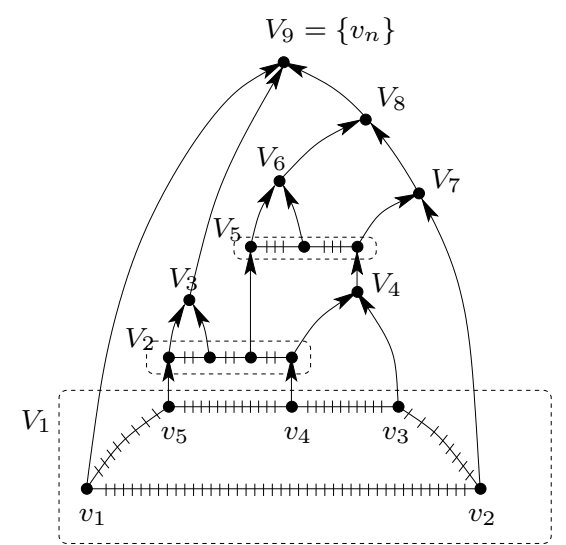

(a)

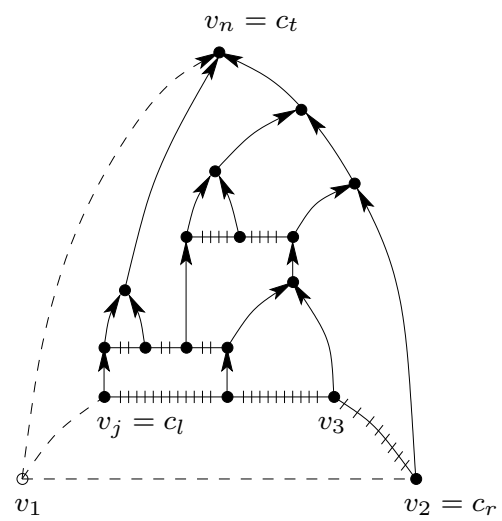

(b)

Figure 1: (a) Canonical orientation of $G$. (b) $G-v_{1}$ is dart-shaped. Undirected edges are hashed.

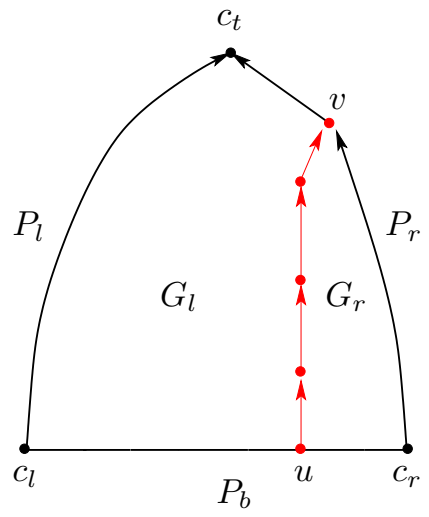

(a)

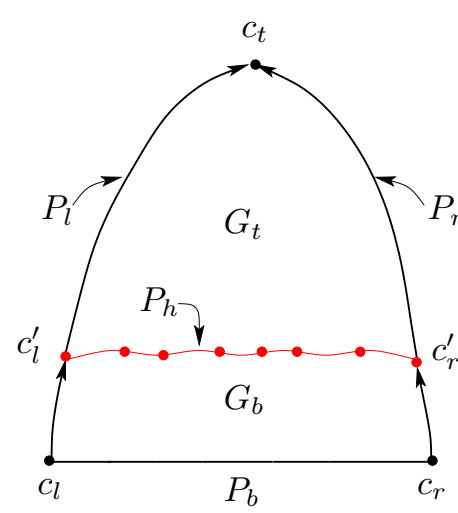

(b)

Figure 2: (a) Vertical decomposition of $G$ into two dart-shaped graphs. (b) Horizontal decomposition of $G$ into a dart shaped graph and a face.

Proof: The proof has two cases, leading to two different splits.

Case (1): $P_{b}$ has at least two edges. Pick any vertex $u \neq c_{l}, c_{r}$ in the interior of $P_{b}$. Follow the vertical path starting from the outgoing edge of $u$. This path is not a cycle because $G$ is acyclic, and it is unique since every vertex $\neq c_{t}$ has out-degree one. Also, the path must reach some vertex $\neq c_{l}, c_{r}, c_{t}$ on $P_{l}$ or $P_{r}$ since vertices on $P_{b}$ have in-degree 0 and $c_{t}$ has in-degree 2 and its incident edges are one on $P_{l}$ and the other on $P_{r}$. Let $v$ be the first vertex on $P_{l} \cup P_{r}$ that is on this path, and let $P_{v}$ be the path from $u$ to $v$. $P_{v}$ divides $G$ into two subgraphs, and it is not hard to verify that they are dart-shaped.

Case (2): $P_{b}$ is an edge $\left(c_{l}, c_{r}\right)$. By condition (D1), $\left(c_{l}, c_{r}\right)$ is undirected. Consider the interior face of $G$ adjacent to $\left(c_{l}, c_{r}\right)$. By (D2), it consists of the edge $\left(c_{l}, c_{r}\right)$, a vertical path $P_{1} \subseteq P_{l}$ (since every vertex has only one outgoing edge) starting at $c_{l}$ and ending at some vertex $c_{l}^{\prime}$, a vertical path $P_{2} \subseteq P_{r}$ starting at $c_{r}$ and ending at some vertex $c_{r}^{\prime}$, and (maybe) a horizontal path $P_{h}$ from $c_{l}^{\prime}$ to $c_{r}^{\prime}$. We know that $\left(c_{l}, c_{r}\right)$ cannot be this horizontal path because $c_{l}$ and $c_{r}$ are in $P_{b}$ and hence 
have no incoming edges.

If $P_{h}$ is empty, then $c_{l}^{\prime}=c_{r}^{\prime}$, which (since $P_{l}$ and $P_{r}$ are interior vertex-disjoint) implies $c_{l}^{\prime}=$ $c_{r}^{\prime}=c_{t}$ and all of $G$ is one interior face, a contradiction. So $P_{h}$ is non-empty, and it splits $G$ into the face containing $\left(c_{l}, c_{r}\right)$ and the subgraph $G_{t}$ containing $c_{t}$. It is not hard to see that $G_{t}$ is dart-shaped, which proves the result.

\section{Algorithm for orthogonal cartograms}

We first provide an outline of our algorithm to create orthogonal cartograms. It has the following steps: (1) Compute the canonical order of $G$ and the partial edge orientation. (2) Let $G^{\prime}=G-v_{1}$; $G^{\prime}$ is dart-shaped. (3) Split $G^{\prime}$ into two dart-shaped graphs $G_{1}$ and $G_{2}$. (4) Draw $G_{1}$ and $G_{2}$ separately (recursively) within a prescribed shape. (5) Combine the drawings of $G_{1}$ and $G_{2}$ into a single drawing.

The difficulty of this algorithm lies in in combining the drawings of $G_{1}$ and $G_{2}$. These two graphs share vertices, so if we draw $G_{1}$ first, this forces some of the vertices to be at fixed locations in the drawing of $G_{2}$. Since our algorithm works recursively, we must allow to fix the positions of some vertices on the outer-face of the graph. But then it is not possible to split the drawing region into simple regions, such as rectangles.

Therefore, we use a significantly more complex shape, which we call a T-staircase, defined formally in Section 4.1. In order to draw subgraphs in it recursively, we need to break it apart into smaller T-staircases; we discuss this in Section 4.2. In Section 4.3 we describe exactly where on a T-staircase the vertices of $G^{\prime}$ can be fixed, so that $G^{\prime}$ can be drawn with correct face areas inside any T-staircase. We call this a correct pinning. Then we combine everything together and explain the choice of T-staircase in the outermost recursion in Lemma 4.3.

\section{$4.1 \quad$ T-staircases}

Definition $2 A$ T-staircase is an $x$-monotone orthogonal polygon for which the upper chain consists of just one edge (the top side) and the lower chain consists of a descending staircase (the left curve), one horizontal edge (the base) and an ascending staircase (the right curve). Furthermore, all corners of the polygon except the two bottommost ones are within distance $\frac{1}{4 t}$ from the top, where $t$ is the length of the top side.

See Figure 3a for an example. The top e-region is the topmost region inside the T-staircase with height $\frac{1}{4 t}$; it has area at most $\frac{1}{4}$ and by definition contains all corners on the left and right curves. Thus, the segments of these curves outside the top $\varepsilon$-region are straight vertical lines. The left and right stairs are the portion of the left and right curves inside the top $\varepsilon$-region and the left and right sides are the segments of the left and right curves outside the top $\varepsilon$-region.

Let $h$ be the height of the left and right curves. The left/right $\varepsilon$-region is a $\frac{1}{4 h} \times h$ rectangle adjacent to the left/right side, and inside the T-staircase. Then, the top, left and right $\varepsilon$-regions together have area at most $\frac{3}{4}$. We use the $\varepsilon$-regions to place all necessary bends. Since the area of these regions is smaller than the area of any face (recall that face areas are integers), it is possible to include any portion of the $\varepsilon$-regions in any face and still be able to draw it with correct area.

The allowed segment of the top side is the segment starting at the $x$-coordinate of the right side of the left $\varepsilon$-region and ending at the $x$-coordinate of the left side of the right $\varepsilon$-region. We 
will later see that vertices on the top side of a T-staircase will only be assigned to points along the allowed segment. For any T-staircase $T$, denote its area by $A(T)$.

\subsection{Decomposing T-staircases}

The idea now is to break any dart-shaped graph $G$ into two pieces (as in Theorem 1), and place the pieces in a T-staircase $T$ recursively. To do so, we first must argue that $T$ can be divided into two T-staircases suitably, even if the endpoints of the dividing line are restricted in their location. Let a pinning point be a point that is either on the interior of a vertical segment of the left or right stairs, or on a reflex corner of the left or right stairs.

We now give two lemmas for how to divide a T-staircase; they mirror (and will be applied to) the two different cases of how to divide a dart-shaped graph.

Lemma 4.1 Let $T$ be a T-staircase and let $1 \leq A \leq A(T)-1$. Then we can divide $T$ with an orthogonal path $l$ into two T-staircases of area $A$ and $A(T)-A$. Moreover, we can restrict $l$ as follows:

- $l$ begins at a (pre-specified) pinning point $v$, or at an (un-specified) point on the allowed segment of $T$.

- $l$ ends at an (un-specified) point on the base of $T$.

- l has at most one bend.

Proof: Assume $l$ is restricted to begin at a pinning point $v$, which by symmetry we assume to be on the left stairs. Draw a horizontal line from $v$ to some $x$-coordinate $X$ (to be determined later) that is above the base; this is feasible since $v$ is at a pinning point. Continue vertically to the base from there. See Figure 3b. If $l$ is instead restricted to begin at the allowed segment, then simple make it a vertical line segment at $x$-coordinate $X$ (to be determined.) See Figure 3c.

To see that a suitable value $X$ exists, use the mean value theorem. If $X$ is at the right side of the left $\varepsilon$-region, then the area to the left of $l$ is $\leq \frac{3}{4}<A$, so this $X$ was too small. If $X$ is at the left side of the right $\varepsilon$-region, then the area to the right of $l$ is $\leq \frac{3}{4}<A(T)-A$, so this $X$ was too big. By the mean value theorem, hence a suitable $X$ exists.

It is straightforward to verify that the two resulting shapes are indeed T-staircases.

Lemma 4.2 Let $T$ be a $T$-staircase and let $1 \leq A \leq A(T)-1$. Then we can divide $T$ with an orthogonal path $l$ into a T-staircase $T^{\prime}$ of area $A$ containing the allowed segment and a face $B$. Moreover, we can restrict $l$ as follows:

- $l$ begins at a pinning point on the left stairs, or at the left endpoint of the allowed segment.

- $l$ ends at a pinning point on the right stairs, or at the right endpoint of the allowed segment.

- $l$ has at most 4 bends.

Proof: Assume first that the endpoints of $l$ are required to be the pinning points $u$ and $v$ on the left/right stairs. We usually cannot draw a straight line from $u$ to $v$ because their positions are fixed, and the line between them may not be horizontal and/or may not create an area of appropriate size. Thus, we connect $u$ to $v$ with a path $l$ with four bends, as follows: 


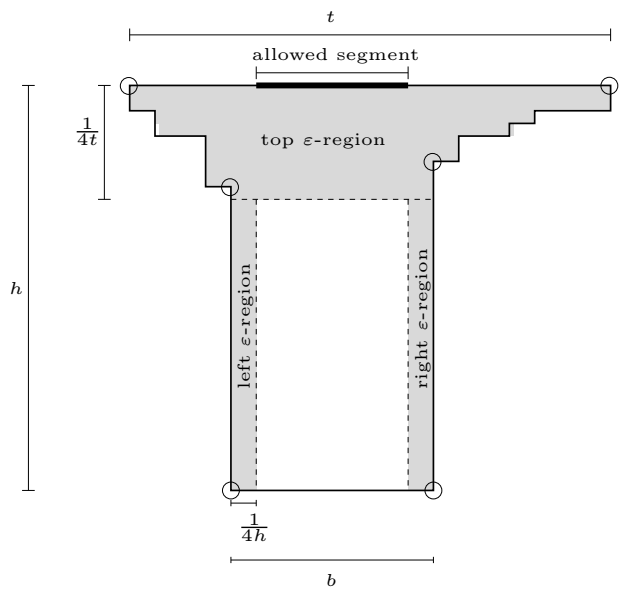

(a)

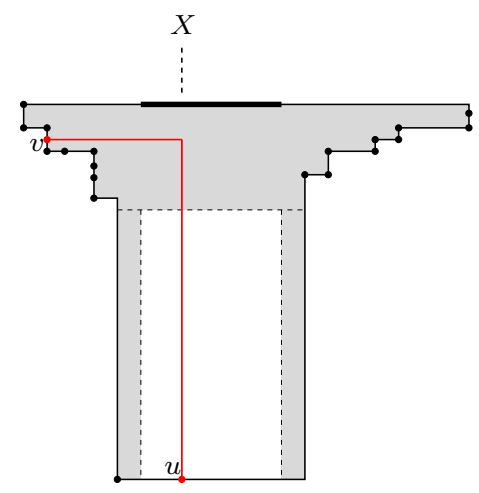

(b)

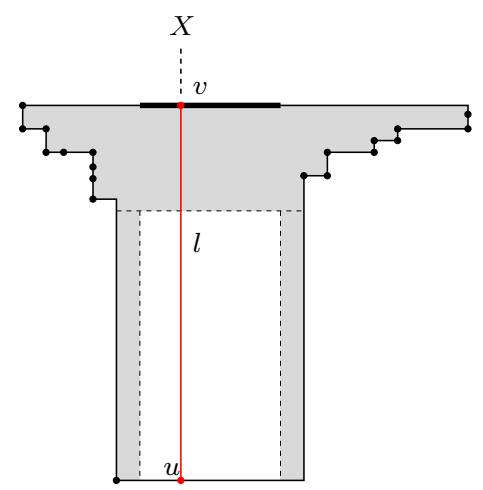

(c)

Figure 3: (a) Example of a T-staircase. (b) and (c) Division of $T$ into two T-staircases with prescribed areas. Figures are not to scale (the height of the $\varepsilon$-regions is hugely exaggerated.)

- Draw a horizontal line segment $S_{1}$ from $u$ to the right until the boundary of the left $\varepsilon$-region.

- Draw a horizontal line segment $S_{5}$ from $v$ to the left until the boundary of the right $\varepsilon$-region.

- Segments $S_{2}\left[S_{4}\right]$ attach to $S_{1}\left[S_{5}\right]$ and go downward until some $y$-coordinate $Y$ (to be determined.)

- Segment $S_{3}$ is a horizontal segment at $y$-coordinate $Y$ that connects $S_{2}$ and $S_{4}$.

The other cases of restrictions on endpoints of $l$ are done similarly, except that we omit segment $S_{1}$ and/or $S_{5}$. See Figure 4 for an example.

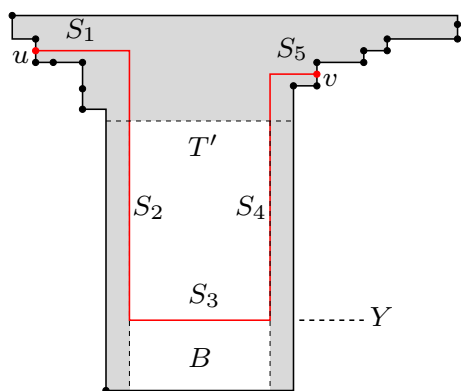

(a)

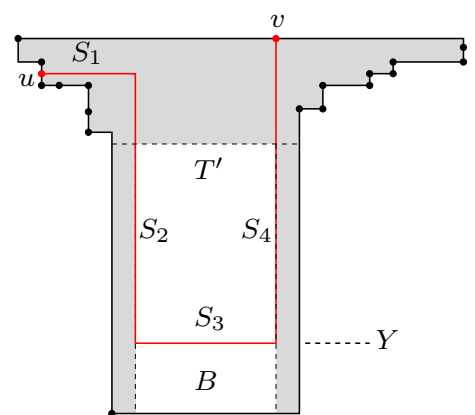

(b)

Figure 4: Example of $B$ and $T^{\prime}$ when (a) both ends of $l$ are at pinning points, and (b) exactly one end of $l$ is at a pinning point.

In any of the cases above, we can see that the area $T^{\prime}$ above $l$ is a T-staircase since its corners (except the bottommost ones) are in the top $\varepsilon$-region. It remains to argue that a suitable $Y$ exists. This is again done with the mean value theorem: $Y=0$ is too small since the area below $l$ would have area $\leq \frac{3}{4}$, but $Y$ at the bottom of the top $\varepsilon$-region is too big, since the area above $l$ would have area $\leq \frac{3}{4}$. So a suitable $Y$ exists. 


\subsection{Pinning dart-shaped graphs to T-staircases}

Now, we will define a condition that guarantees that a dart-shaped graph with prescribed interior face areas can be drawn inside a T-staircase:

Definition 3 Let $G$ be a dart-shaped graph and $T$ be a T-staircase. A partial assignment of outerface vertices of $G$ to points on the boundary of $T$ is called a correct pinning of $G$ to $T$ if the following constraints are satisfied:

C1. The points assigned to vertices correspond to the order of vertices along the outer-face of $G$.

C2. Any vertex $v \in P_{l} / P_{r}$ with $\operatorname{deg}(v)=3$ is either assigned to a pinning point on the left/right stairs, or could be assigned to a point on the allowed segment such that the order of vertices is respected.

C3. Any corner of $T$ has a vertex assigned to it, with at most 6 exceptions: the endpoints of the top side, the endpoints of the base, and the bottommost corner of the left and right stairs. These points are circled in Figure $3 a$.

C4. If there is a bend at the bottommost corner of the left or right stairs, then there are no vertices assigned to the vertical segment below it except (perhaps) at the endpoints of the base.

Lemma 4.3 Let $G$ be a dart-shaped graph that is pinned correctly to a T-staircase $T$ of area $A(G)$. Then $G$ has a drawing inside $T$ that respects the pinned vertices and the given face areas.

Proof: Nothing is to show if $G$ has only a single interior face, since the correct pinning ensures that fixed vertices are drawn in order, and unpinned vertices can be added suitably. So assume that $G$ has at least two interior faces. By Theorem $1 G$ can be split into smaller graphs, either with a vertical path or a horizontal path. We now distinguish two cases, depending on the type of split.

Case (1): Assume $G$ can be divided into two dart-shaped graphs $G_{l}$ and $G_{r}$ by a vertical path from a vertex $u \neq c_{l}, c_{r}$ in $P_{b}$ to a vertex $v \neq c_{l}, c_{r}, c_{t}$ in $P_{l} \cup P_{r}$. Assume that $v \in P_{l}$; the other case is similar. Since $G$ is pinned correctly to $T$ and $\operatorname{deg}(v)=3$, vertex $v$ is either assigned to a pinning point on the left stairs or could be placed on the allowed segment. By Lemma 4.1, we can divide $T$ into two T-staircases $T_{l}$ and $T_{r}$ of area $A\left(G_{l}\right)$ and $A\left(G_{r}\right)$ such that the dividing line $l$ begins at the pinning point of $v$ (or at the allowed segment where we then place $v$ ), and ends at the base (where we then place $u$.)

The interior vertices of $P_{v}$ are pinned to $l$ as follows:

- If $v$ was on the allowed segment, then $l$ is a vertical segment. Pin all vertices of $P_{v}$ in order on $l$ inside the top $\varepsilon$-region. See Figure 5 a.

- If $v$ was at a pinning point (hence $l$ has a bend $b$ ), then let $w$ be the topmost (closest to $v$ ) vertex of degree 3 on $P_{v}$ for which the neighbour that is not on $P_{v}$ belongs to $G_{r}$. If $w$ does not exist, then all vertices in $P_{v}$ remain unpinned (they will be placed on the allowed segment for $G_{l}$, and have degree 2 in $G_{r}$.) If $w$ does exist, then pin it to $b$, and pin all vertices between $w$ and $u$ on $P_{l}$ on the vertical segment of $l$, below $w$, in order and within the top $\varepsilon$-region. The other points of $P_{l}$ remain unpinned and will be on the horizontal segment adjacent to $w$ (which is the allowed segment for $T_{l}$.) See Figure 5b. 
The verification that this is a correct pinning for both subgraphs and both T-staircases is tedious but straightforward, and therefore left to the reader.

Case (2): Assume $G$ can be divided into a dart-shaped graph $G_{t}$ and a single face $G_{b}$ by a horizontal path $P_{h}$ from a vertex $c_{l}^{\prime} \neq c_{l}, c_{t} \in P_{l}$ to a vertex $c_{r}^{\prime} \neq c_{r}, c_{t} \in P_{r}$. Since $G$ is pinned correctly to $T, c_{l}^{\prime}$ and $c_{r}^{\prime}$ may be assigned to a pinning point or could be placed on the allowed segment. By Lemma 4.2 it is possible to draw an orthogonal path $l$ from $c_{l}^{\prime}$ to $c_{r}^{\prime}$ that divides $T$ into a T-staircase $T^{\prime}$ of area $A\left(G_{t}\right)$ and a shape $B$ of area $A\left(G_{b}\right)$. We will not assign any vertices $\neq c_{l}^{\prime}, c_{r}^{\prime}$ to positions in $T^{\prime}$. We again leave to the reader the lengthy but straightforward verification that this indeed pins $G_{t}$ to $T^{\prime}$ correctly.

So in either case, the subgraphs that have more than one interior face have been pinned correctly to a T-staircase of appropriate area. Hence by induction the subgraphs can be drawn while respecting the pinned vertices, and putting the drawings together gives a drawing of $G$.

Theorem 2 Any 3-regular 3-connected planar graph $G=(V, E)$ can be drawn orthogonally with given interior face areas, at most 4 bends per edge, and at most 12 corners per face.

Proof: Compute a canonical order with $V_{1}=\left\{v_{1}, \ldots, v_{j}\right\}$ and $V_{K}=\left\{v_{n}\right\}$ and orient edges accordingly. Then $G^{\prime}=G-v_{1}$ is dart-shaped with corners $c_{l}=v_{j}, c_{r}=v_{2}$ and $c_{t}=v_{n}$ (Lemma 3.1.)

Let $T$ be any rectangle with area $A\left(G^{\prime}\right)$. Clearly $T$ is a T-staircase. Pin $v_{j}$ to the bottom left corner, $v_{2}$ to the bottom right corner, and $v_{n}$ to the left top corner of $T$. Pin all vertices on the vertical path from $v_{j}$ to $v_{n}$ to the left side of $T$, in order, and inside the $\varepsilon$-region. It is straightforward to verify that $G^{\prime}$ is pinned correctly to $T$.

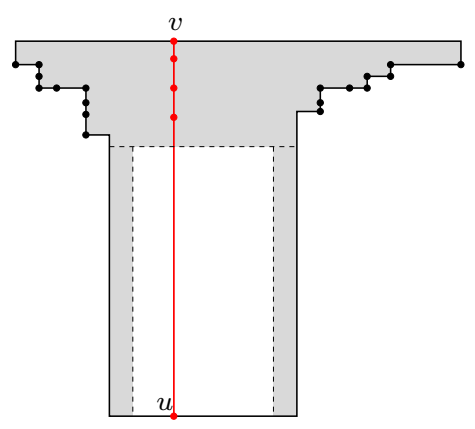

(a)

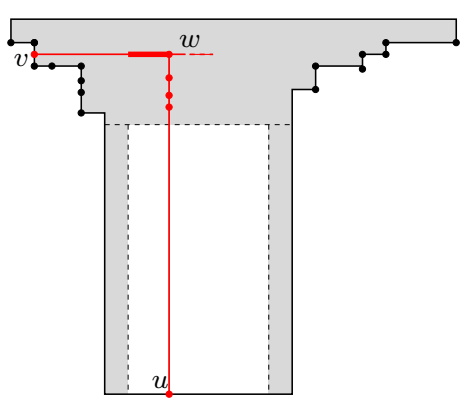

(b)

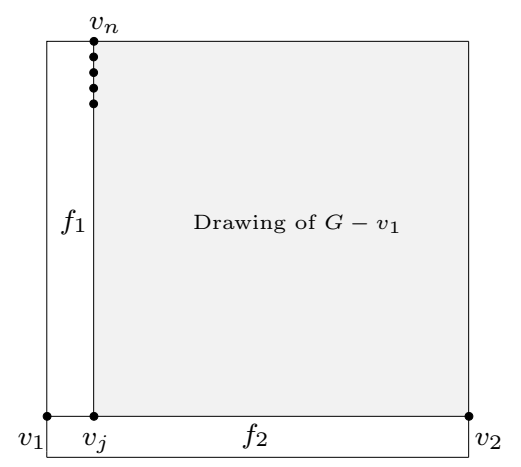

(c)

Figure 5: (a) Pinning vertices when dividing the T-staircase vertically. (b) Pinning vertices when dividing the T-staircase with one bend, and vertex $w$ exists. (c) The base case: Pinning $v_{2}$ and the vertices on $P_{l}$, and how to add $v_{1}$.

By Lemma 4.3, we can recursively draw $G^{\prime}$ inside $T$ respecting the face areas. Any edge is part of a dividing path created during the algorithm, and hence has at most 4 bends.

Now we argue that each interior face of $G^{\prime}$ has at most 12 corners, and do this by arguing that it has at most 4 reflex corners. Observe that such corners are necessarily at bends, since vertices $\neq v_{1}, v_{2}, v_{n}$ have degree 3 , and $v_{1}, v_{2}, v_{n}$ do not form reflex corners for interior faces. 
Recall that any T-staircase has at most 2 bends that are at reflex corners if its graph is pinned correctly to it. Hence any face reached in the base case of the recursion has at most 2 reflex corners. The only kind of face not reached in the base case is the face $B$ below the dividing path $l$ that we create in Lemma 4.2. This dividing path $l$ adds at most 2 reflex corners to the face below, so such a face has at most 4 reflex corners. Either way, each face has at most $k \leq 4$ reflex corners. Since it has $k+4$ convex corners, the total number of corners is at most 12 .

Thus $G^{\prime}$ is now drawn inside the rectangle with at most 4 bends per edge and 12 corners per face. To draw $G$, we need to add the vertex $v_{1}$ and its three incident edges, and it is easy to do so while respecting areas and with 2 bends in those edges; see Figure $5 c$.

\section{$5 \quad$ Remarks}

We would like to end the paper with numerous remarks and pointers for future work.

- Following our construction, it is easy to see that all coordinates are rational if we choose the rectangle for the outer-most recursion, and the "free" coordinate for pinning vertices to be rational. However, it remains open how small the rational coordinates are, i.e., what grid-size would be needed if we scaled them to be integers. We do not expect a polynomial bound here, but do the coordinates satisfy, say, $O\left(n ! 2^{n}\right)$ if $A \equiv 1$ ? Or can we at last bound the minimum edge-segment length or minimum feature size?

- While the coordinates in our drawings depend on the prescribed areas, the shape of the drawing, i.e., which edge bends in which direction how often, is independent of it. Hence our drawings are area-universal: For any assignment of areas, we can change the length of the edges only and obtain a planar drawing that respects the area assignment.

- It is easy to compute the shape of our drawing in linear time, following the steps of the recursion. However, computing the actual coordinates is more complicated. The bottleneck is the computation of $X$ in Lemma 4.1. Computing this using the smaller of the subgraphs leads to an $O(n \log n)$ time, amortized over all vertices (details are omitted.) Reducing this to $O(n)$ remains an open problem.

- Our algorithm works for graphs with maximum degree 3. Extending it to higher degrees would require generalizing dart-shaped graphs (which is fairly straightforward: the bottom horizontal path must be allowed to vanish.) But we have not been able to generalize a Tstaircase such that many vertices may be at reflex vertices (as required if they have degree 4), without creating too many bends, and allowing for easy division. It is known [15] that every graph with maximum degree 4 has an orthogonal drawings respecting given face areas, but it remains open whether we can additionally demand $O(1)$ corners per face always.

- Our algorithm was designed for 3-connected graphs. It is possible to extend this result to graphs with less connectivity by introducing extra vertices and edges until the graph is 3connected, and removing them in the final drawing. However, with this approach the number of corners per face could be arbitrarily large. Can we instead explicitly maintain and combine 3-connected components to obtain a small constant number of corners per face?

- Finally, area-respecting drawings could also be studied in the hexagonal or octagonal grid. Can such grids be used to reduce the number of corners to less than 12 ? 


\section{References}

[1] Marek Chrobak and Shin ichi Nakano. Minimum-width grid drawings of plane graphs. In Graph Drawing (GD '94), Lecture Notes in Computer Science, volume 894, pages 104-110. Springer, 1994.

[2] Marek Chrobak and Goos Kant. Convex grid drawings of 3-connected planar graphs. Int. Journal of Computational Geometry and Applications, 7(3):211-223, 1997.

[3] Mark de Berg, Elena Mumford, and Bettina Speckmann. On rectilinear duals for vertexweighted plane graphs. In Proc. 13th International Symposium on Graph Drawing (GD'05), Lecture Notes in Computer Science, volume 3843, pages 61-72. Springer, 2006.

[4] Mark de Berg, Elena Mumford, and Bettina Speckmann. Optimal BSPs and rectilinear cartograms. In GIS '06: Proceedings of the 14th annual ACM international symposium on Advances in geographic information systems, pages 19-26, New York, NY, USA, 2006. ACM.

[5] Mark de Berg, Elena Mumford, and Bettina Speckmann. On rectilinear duals for vertexweighted plane graphs. Discrete Mathematics, 309(7):1794-1812, 2009.

[6] Mark de Berg, Elena Mumford, and Bettina Speckmann. Optimal bsps and rectilinear cartograms. Int. J. Comput. Geometry Appl., 20(2):203-222, 2010.

[7] H. de Fraysseix, J. Pach, and R. Pollack. How to draw a planar graph on a grid. Combinatorica, 10:41-51, 1990.

[8] V. Dujmović, D. Eppstein, M. Suderman, and D. Wood. Drawings of planar graphs with few slopes and segments. In Computational Geometry Theory and Applications, volume 38, pages 194-212. Springer, 2005.

[9] D. Eppstein, E. Mumford, B. Speckmann, and K. Verbeek. Area-universal rectangular layouts. In Proc. 25th ACM Symposium on Computational Geometry (SoCG), pages 267-276, 2009.

[10] I. Fáry. On straight line representation of planar graphs. Acta. Sci. Math. Szeged, 11:229-233, 1948.

[11] Jim Geelen, Anjie Guo, and David McKinnon. Straight line embeddings of cubic planar graphs with integer edge lengths. Journal of Graph Theory, 58(3):270-274, 2008.

[12] Roland Heilmann, Daniel A. Keim, Christian Panse, and Mike Sips. Recmap: Rectangular map approximations. In Info Vis 2004, pages 33-40. IEEE, 2004.

[13] G. Kant. Drawing planar graphs using the canonical ordering. Algorithmica, 16(1):4-32, 1996.

[14] Goos Kant and Xin He. Regular edge labeling of 4-connected plane graphs and its applications in graph drawing problems. Theor. Comput. Sci., 172(1-2):175-193, 1997.

[15] Akifumi Kawaguchi and Hiroshi Nagamochi. Orthogonal drawings for plane graphs with specified face areas. In Proc. 4th Int. Conf. on Theory and Applications of Models of Computation, Lecture Notes in Computer Science., volume 4484, pages 584-594. Springer, 2007. 
[16] Wouter Meulemans, André van Renssen, and Bettina Speckmann. Area-preserving subdivision schematization. In GIScience, volume 6292 of Lecture Notes in Computer Science, pages 160174. Springer, 2010.

[17] Takao Nishizeki and Md. Saidur Rahman. Planar Graph Drawing (Lecture Notes Series on Computing). World Scientific Publishing Company, 2004.

[18] Md. Saidur Rahman, Kazuyuki Miura, and Takao Nishizeki. Octagonal drawings of plane graphs with prescribed face areas. Comput. Geom. Theory Appl., 42(3):214-230, 2009.

[19] Erwin Raisz. The rectangular statistical cartogram. Geographical Review, 24(3):292-296, 1934.

[20] Gerhard Ringel. Equiareal graphs. Contemporary Methods in Graph Theory. BI Wissenschaftsverlag, pages 503-505, 1990.

[21] Lesvia Elena Ruiz Velázquez. Drawing planar graphs with prescribed face areas. Master's thesis, University of Waterloo, 2010. http://uwspace.uwaterloo.ca/bitstream/10012/5481/1/RuizVelazquezLesviaElena.pdf.

[22] W. Schnyder. Embedding planar graphs on the grid. In SODA '90: Proceedings of the first Annual ACM-SIAM Symposium on Discrete Algorithms, pages 138-148, Philadelphia, PA, USA, 1990. Society for Industrial and Applied Mathematics.

[23] S. Stein. Convex maps. American Mathematical Society, 2:464-466, 1951.

[24] Carsten Thomassen. Plane cubic graphs with prescribed face areas. Combinatorics, Probability \& Computing, 1:371-381, 1992.

[25] Peter Ungar. On Diagrams Representing Maps. Journal of the London Mathematical Society, s1-28(3):336-342, 1953.

[26] M. van Kreveld and B. Speckmann. On rectangular cartograms. In Proc. 12th European Symposium on Algorithms (ESA), Lecture Notes in Computer Science., volume 3221, pages 724-735. Springer, 2004.

[27] K. Wagner. Bemerkungen zum Vierfarbenproblem. Jahresbericht der Deutschen Mathematiker-Vereinigung, 46:26-32, 1936. 